\section{Poly(dA:dT) make it and break it}

DNA replication stress causes chromosomal instability, especially at genomic regions termed fragile sites, the molecular basis of which is unclear. Tubbs et al. show that fragile-site instability is associated with replication-fork collapse and formation of DNA doublestranded breaks (DSBs) at poly(dA:dT) tracts, and that poly(dA:dT) tracts are also enriched and have a similar effect at strong origins of replication.

To investigate replication-related DSB formation, the authors treated mouse and human cells with hydroxyurea, which causes replication elongation stress, and mapped DSB breakpoints genome-wide at nucleotide resolution. Many DSBs overlapped early-replicating fragile sites (ERFSs), and most of these DSBs overlapped replication initiation zones and formed in a replication-dependent manner, indicating that ERFSs are regions of fork collapse at active replication origins. DSBs in replication initiation zones were bordered by poly $(\mathrm{dA})$ on the 3 ' side and poly $(\mathrm{dT})$ on the $5^{\prime}$ side, suggesting that poly(dA:dT) sequences near replication origins are hot spots of replication-fork stalling and collapse.

Following milder hydroxyurea treatment (and also in untreated cells), DSBs clustered also at AT-rich sequences at late-replicating common fragile sites (CFSs). The majority of these DSBs were within $20 \mathrm{bp}$ of poly(dA:dT) tracts. Thus, replication-fork collapse at poly(dA:dT) tracts during replication stress probably contributes to chromosomal fragility at both ERFSs and CFSs.

The two ends (sides) of the DSBs were not symmetrical. Breaks at poly(dT) tracts reproducibly occurred at nucleotide position 10; indeed, the levels of DNA replication precipitously dropped at $\mathrm{dT}_{10}$ at left-moving replication forks and at $\mathrm{dA}_{10}$ at right-moving forks. Thus, forks under stress encountering a leading-strand template of poly(dT) stall

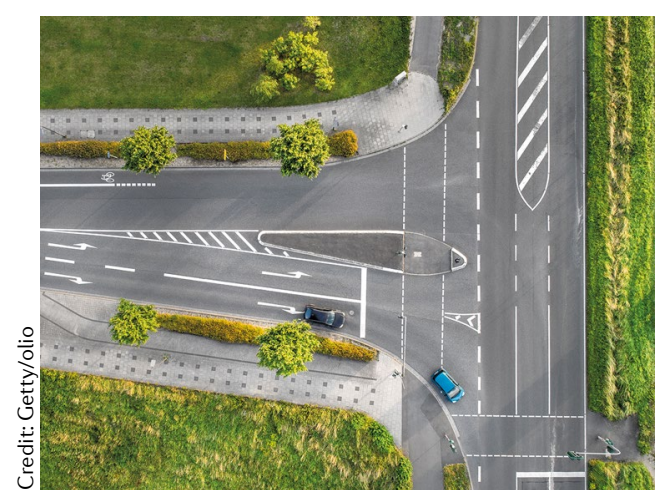

around position $\mathrm{T}_{10}$ and produce well-defined

The breakpoints at the poly $(\mathrm{dA})$ tracts occurred within long (250-400 nucleotide) stretches of unwound, single-stranded DNA (ssDNA), which is probably formed by the physical uncoupling of the unperturbed replication helicase and the stalled polymerase. In support of poly(dA) breakpoints occurring within ssDNA, the ssDNA binding protein replication protein A (RPA) was found to bind only the poly(dT) strand, thereby possibly exposing the poly $(\mathrm{dA})$ tract to attack by nucleases and to DSB formation at

Replication origins in yeast are characterized by high AT content and nucleosome depletion, which could facilitate DNA unwinding and thus origin firing. By contrast, replication origins are poorly characterized in metazoans owing to their functional redundancy and lack of sequence consensus. The authors precisely mapped thousands of strong (frequently firing) replication origins in human and mouse cells, and found high AT content and considerable depletion of nucleosomes at the peaks of replication initiation zones. Thus, replication origin selection could be a conserved and regulated process.

Interestingly, similarly to fragile sites, sequences flanking these replication initiation peaks also exhibited asymmetrical nucleotide distribution, with higher poly $(\mathrm{dA})$ content on the $3^{\prime}$ side and higher poly $(\mathrm{dT})$ content on the $5^{\prime}$ side. Furthermore, hydroxyurea-induced DSBs were most frequent at poly $(\mathrm{dA}: \mathrm{dT})$ tracts located several hundred base pairs from the replication initiation peaks, raising the possibility that origin firing is naturally followed by 'pausing' at poly (dA:dT) tracts prior to the establishment of processive replication elongation.

In summary, ERFSs and CFSs share a common mechanism of fragility, which is based on fork stalling and collapse at poly(dA:dT) tracts. Such sequences also characterize strong replication origins, where they can cause fork collapse and DSBs. The role of poly(dA:dT) tracts in replication initiation could explain their high conservation and abundance in eukaryotic genomes, despite their inherent susceptibility to DSB formation.

Eytan Zlotorynski

ORIGINAL ARTICLE Tubbs, A. et al. Dual roles of poly(dA:dT) tracts in replication initiation and fork collapse. Cell https://doi.org/10.1016/j.cell.2018.07.011 (2018) FURTHER READING Glover, T. W. et al. Fragile sites in cancer: more than meets the eye. Nat. Rev. Cancer 17, 489-501 (2017) breakpoints. random positions.

\section{GENOME EDITING}

\section{Not so CRISP(R)}

The CRISPR-Cas9 system is thought to be a reasonably specific method for genome editing. But Bradley and colleagues now report that CRISPRCas9 induces extensive on-target mutagenesis in mouse and human cells, calling for greater caution when using it in clinical contexts and stressing the need for comprehensive genomic analyses before edited cells can be used in patients.

The authors analysed the allelic diversity induced by the introduction of Cas9 and single-guide RNAs that target introns and exons of PigA (an X-linked locus) in mouse embryonic stem cells (ESCs), as targeting the introns also led to the loss of PigA. Sequencing of PigA-deficient single-cell clones revealed that, in addition to creating the expected insertions and deletions (indels) of $<50 \mathrm{bp}, \mathrm{CRISPR}-\mathrm{Cas} 9$ generated deletions of $>250 \mathrm{bp}$ to $6 \mathrm{~kb}$ in more than $20 \%$ of alleles. Moreover, single-nucleotide polymorphisms, indels and large deletions and insertions that were non-contiguous with the Cas9 cut site were identified; such mutations would be missed if the analysis was limited to the vicinity of the cleavage site, as is often the case. In some cases, changes in parts of the genome distant from the target site were also found.

By editing and analysing other loci in different cell lines - immortalized human retinal pigment epithelial cells and progenitor cells isolated from mouse bone marrow - the authors confirmed that the extensive ontarget damage, which is associated with DNA repair, is not an intrinsic property of mouse ESCs.

Thus, CRISPR-Cas9 can induce extensive on-target genomic rearrangements that could potentially lead to pathogenic lesions in clinically relevant cell types.

Kim Baumann

ORIGINAL ARTICLE Kosicki, M. et al. Repair of double-strand breaks induced by CRISPR-Cas 9 leads to large deletions and complex rearrangements. Nat. Biotechnol. 36, 765-771 (2018) FURTHER READING Dominguez, A. A. et al. Beyond editing: repurposing CRISPR-Cas 9 for precision genome regulation and interrogation. Nat. Rev. Mol.Cell Biol. 17, 5-15 (2016) 